\title{
MEDIDAS SUSTITUTIVAS A LA PENA DE PRIVACIÓN DE LA LIBERTAD
}

\author{
Rodrigo Escobar GiL* \\ Universidad JaVeriana, Colombia \\ rodrigoescg@hotmail.com
}

\section{INTRODUCCIÓN}

El objetivo de esta breve disertación consiste en plantear unas reflexiones que permitan formular un diseño y configuración de las medidas alternativas o sustitutivas a la privación de la libertad, inspiradas en valores y principios humanistas y democráticos, y de esta manera explorar caminos distintos a la tradicional pena de prisión que resulten efectivos e idóneos para cumplir con la función resocializadora de la pena.

En primer lugar abordaremos el tema de los fines del Derecho Penal y de las penas, lo que nos permitirá contextualizar nuestro tema de estudio. En segundo lugar, analizaremos brevemente la naturaleza y características de la pena privativa de la libertad. Posteriormente, examinaremos las medidas sustitutivas a la privación de la libertad, precisando sus funciones, particularidades, instrumentos internacionales que las consagran y ejemplos de algunas de ellas. Finalmente, cerraremos esta exposición con unas breves conclusiones y reflexiones sobre la adopción de las medidas sustitutivas a la privación de la libertad en los diferentes ordenamientos penales nacionales.

\section{FINES DEL DERECHO PENAL Y DE LAS PENAS}

El Derecho Penal constituye la máxima expresión del poder sancionatorio del Estado, que se dirige a la protección de los bienes jurídicos y a la preservación de un orden social justo, cuya aplicación, como ya dijimos, atiende al carácter de ultima ratio, en atención a la necesidad de privilegiar la libertad personal, la dignidad humana y demás derechos e intereses iusfundamentales reconocidos por el Derecho Internacional como inherentes a todas las personas.

* Abogado de la Universidad Javeriana y Doctor en Derecho de la Universidad Complutense de Madrid. 
De esta forma, no obstante que las sociedades acuden como medio de control social a la imposición de penas, previa definición legal y con observancia de las garantías procesales pertinentes, lo cierto es que a estas solo puede acudirse como último recurso, en atención a que el derecho penal en los Estados democráticos solo tiene justificación como la ultima ratio que se ponga en actividad para garantizar la pacífica convivencia de los asociados.

En este contexto de restricción o moderación en el ejercicio del poder punitivo del Estado, vale la pena recordar que la pena que se impone con motivo del ejercicio de conductas que afectan gravemente los bienes jurídicamente tutelados, cumple, en primer lugar, una función preventiva general para que las personas se abstengan de realizar el comportamiento delictivo so pena de incurrir en la imposición de sanciones y, en segundo lugar, una función preventiva especial mediante la imposición de la pena en concreto, con la represión que implica castigar efectivamente las actuaciones delictivas con el objeto de evitar que aquel que haya cometido un acto ilícito vuelva a tener tal actitud en el futuro.

Las penas, sin embargo, no solo deben satisfacer fines preventivos o represivos, sino que deben comportar un aspecto estabilizador de suerte que se presenten como socialmente necesarias para mantener las estructuras fundamentales del conglomerado social. De esta forma, si bien las penas deben procurar defender a la comunidad de quien ha trasgredido los intereses jurídicos protegidos, lo cierto es que también deben procurar el respeto de la dignidad y los derechos de los infractores, mediante la imposición de penas razonables, adecuadas, necesarias y proporcionales y a través del ofrecimiento de alternativas a su comportamiento desviado, a fin de que se obtenga la reinserción social.

Sin perjuicio de las discusiones académicas que pueda haber sobre el particular, a la luz de los tratados internacionales y de la legislación más representativa de los Estados democráticos contemporáneos, que se fundan en el valor superior de la dignidad de la persona humana, es dado afirmar que uno de los fines primordiales de la pena, sin perjuicio de sus propósitos preventivos y retributivos, es la resocialización del condenado. En efecto, es posible señalar que solo son compatibles con los derechos humanos las penas que tiendan a la resocialización del infractor de la ley penal, mediante su incorporación a la sociedad como un sujeto que la engrandece.

Conforme a lo anterior, la política criminal y la aplicación judicial de las penas por parte de los Estados deben atender indefectiblemente a los principios y criterios de razonabilidad, idoneidad, necesidad y proporcionalidad, de suerte que se garantice que la aplicación de una sanción penal, que por definición restringe el ejercicio de derechos inherentes al condenado, sirva para la preservación de la convivencia armónica y pacífica de los asociados ${ }^{1}$ y

\footnotetext{
No solo en cuanto ella por su poder disuasivo e intimidatorio evite la comisión de conductas delictuales, o por lo menos las disminuya, sino también en cuanto, ya cometidas por alguien, su imposición reafirme la decisión del Estado de conservar y proteger los derechos objeto de tutela jurídica.
} 
cumpla además la función de permitir la reincorporación del autor de la conducta punible a la sociedad ${ }^{2}$ con el menor sacrificio de derechos del condenado.

De esta forma, es importante señalar que si una pena se revela irrazonable, inadecuada o desproporcionada, la misma deviene inútil y, en consecuencia, su imposición supone una notoria injusticia. Sobre el particular, como precisaremos más adelante, es importante señalar que si los fines de una sanción penal se pueden alcanzar con medidas menos lesivas de la dignidad humana y, en general de los derechos de las personas, es necesario dar aplicación a tales medidas que armonizan las funciones y objetivos de las penas y los derechos de los condenados. Así, en el marco de los Estados democráticos, la pena, como instrumento adecuado para servir a los fines de prevención, retribución y resocialización, debe ser razonable, idónea y proporcionada; lo que significa que si los mismos fines pueden lograrse por otros medios sancionatorios, debe preferirse el menos severo (pues el más restrictivo dejaría de ser necesario y útil), en aras de garantizar la dignidad del condenado.

Es por ello que a la luz de los principios y criterios de razonabilidad, idoneidad, necesidad y proporcionalidad, es dado a los titulares de la política criminal de los Estados y a los operadores jurídicos, especialmente a los jueces que tienen la función de aplicar la ley penal, determinar que si un condenado, bajo determinadas condiciones y circunstancias, no necesita de la privación física de la libertad para readaptarse a la sociedad, debe brindársele la oportunidad de cumplir con su condena mediante mecanismos que, sin dejar de ser eficaces, comporten una menor aflicción.

\section{LA PENA PRIVATIVA DE LA LIBERTAD}

La pena privativa de la libertad, entendida como la restricción total de la libertad personal del sujeto condenado, es la pena preponderante en el Estado moderno desde varios siglos atrás, y la sanción criminal más común y drástica en los ordenamientos jurídicos de Occidente, a excepción de la pena de muerte que hoy en día se aplica en muy pocos países. La privación de la libertad personal, debido a su naturaleza restrictiva sobre derechos fundamentales, solo debería utilizarse como último recurso para enfrentar conductas delictivas de la más alta gravedad, en otras palabras, cuando sea estrictamente necesaria en vista de la naturaleza y gravedad del delito, la personalidad del delincuente y las necesidad de la convivencia social.

Así entonces, es claro que analógicamente con el espíritu del derecho penal moderno, las penas privativas de la libertad deben utilizarse como ultima ratio, ya que la libertad personal es la regla general, y así ha sido reconocida en todos los instrumentos internacionales. Por ejemplo, el principio III de los "Principios y Buenas Prácticas sobre la Protección de las

De tal manera que pueda, de nuevo, ser parte activa de ella, en las mismas condiciones que los demás ciudadanos en el desarrollo económico, político, social y cultural. 
Personas Privadas de la Libertad en las Américas", adoptado por la CIDH en el año 2008, señala: "Toda persona tendrá derecho a la libertad personal y a ser protegida contra todo tipo de privación de libertad ilegal o arbitraria", y más adelante agrega: "Por regla general, la privación de libertad de una persona deberá aplicarse durante el tiempo mínimo necesario". Así pues, es claro que la restricción de la libertad de una persona es una medida excepcional que solo tiene lugar cuando es absolutamente necesaria, después de haberse cumplido todos los procedimientos legales previstos para imponer tal sanción y, en el mismo sentido, aun cuando resulte procedente la privación de la libertad de una persona, dicha medida solo procederá durante el tiempo mínimo requerido, pues se estima que si ya se ha cumplido con los fines de la pena, no tiene sentido prolongar una pena que afecta el derecho a la libertad.

Este principio referente a la privación de la libertad como ultima ratio, ha sido desarrollado profusamente por la doctrina y jurisprudencia tanto en el sistema interamericano como en el sistema universal de derechos humanos en torno al tema de la detención preventiva como medida cautelar dentro del proceso penal, que tiene como propósito asegurar el desarrollo eficiente de las investigaciones y la comparecencia del procesado al juicio. Al respecto se ha recalcado que el uso de la detención preventiva debe ser excepcional y no puede convertirse en regla general y solo podrá imponerse cuando estén dados los supuestos jurídicos y fácticos y sea necesaria para llevar a buen término el proceso penal. Asimismo, como consecuencia del principio de presunción de inocencia, se exige un límite temporal razonable de la medida.

Ahora bien, diversos instrumentos internacionales, como los "Principios y Buenas Prácticas sobre la Protección de las Personas Privadas de la Libertad en las Américas", se han referido a los derechos de las personas privadas de la libertad y a los requisitos y límites que deben cumplir las penas privativas de la libertad, destacándose la importancia del debido proceso legal, el respeto por la vida, dignidad e integridad física, psicológica y moral de los penados y las finalidades esenciales que deben orientar este tipo de penas, entre las que se encuentran la readaptación social y la rehabilitación personal de los condenados; la resocialización y reintegración familiar; así como la protección de las víctimas y de la sociedad.

Así entonces, dado el gran impacto que tienen sobre los derechos de las personas privadas de la libertad, las penas privativas de la libertad deben estar sujetas a un juicio de proporcionalidad en sentido estricto que demuestre la legalidad, legitimidad, idoneidad, necesidad y proporcionalidad de la pena en sí misma considerada. En cuanto a la legalidad, podemos afirmar entonces, que toda pena privativa de la libertad debe estar previamente consagrada en los ordenamientos jurídicos internos, con una clara precisión de las causas y condiciones en que operará, con sujeción a los límites y requisitos establecidos por los instrumentos internacionales de derechos humanos. Asimismo, el principio de legalidad impone que toda orden que conlleve a una privación de la libertad debe ser emitida por una autoridad competente y mediante una resolución debidamente motivada. 
La legitimidad apunta a que los fines de la pena privativa de la libertad se ajusten teleológicamente a los valores, principios y derechos establecidos en un orden constitucional democrático, como serían la búsqueda efectiva de la convivencia social y de un orden social justo, la resocialización de la persona condenada y la garantía de los derechos de las víctimas, fines comúnmente aceptados a nivel internacional.

El tercer paso hace referencia a la idoneidad de las penas privativas de la libertad para alcanzar los fines propuestos, ya que debe existir una estrecha correlación entre el fin que se pretende alcanzar y la pena que se impone para lograr este cometido, es decir, debe quedar plenamente establecido que la pena privativa de la libertad sea material y socialmente adecuada para la protección de la sociedad, la resocialización de la persona condenada y la garantía de los derechos de las víctimas.

El cuarto paso, y este es el punto que quiero resaltar, indica que la pena privativa de libertad debe ser necesaria. El principio de necesidad de la pena es de vital importancia en el derecho penal, ya que al ser la pena una intervención grave por parte del Estado sobre los derechos humanos de las personas, es imperativo analizar si esta intromisión resulta absolutamente necesaria para cumplir con los fines de la pena, es decir, que no se puede reemplazar por una medida alternativa menos lesiva. Es entonces en este momento en donde se debe evaluar la posibilidad de adoptar medidas sustitutivas a la pena de privación de la libertad, pues si de la evaluación que se hace al principio de necesidad, se concluye que mediante el uso adecuado de otras medidas, distintas a la pena privativa de la libertad, se pueden alcanzar los fines resocializadores de la pena, de protección a la sociedad y de garantía de los derechos de las víctimas, deberá preferirse el uso de estas medidas sustitutivas, pues significa que la restricción de la libertad no es necesaria y estas medidas resultarían menos lesivas sobre los derechos de las personas condenadas penalmente, facilitando su resocialización, al mismo tiempo que se garantiza la protección a la sociedad.

Ahora bien, una vez agotados los cuatro pasos anteriores, es necesario realizar un juico de proporcionalidad propiamente dicho sobre la pena privativa de la libertad, lo que exige que los beneficios de adoptar la medida excedan claramente las restricciones impuestas sobre otros principios y valores constitucionales por la medida, en este caso la libertad personal.

\section{MEDIDAS SUSTITUTIVAS A LA PRIVACIÓN DE LA LIBERTAD}

Las medidas alternativas o sustitutivas a la privación de la libertad son instrumentos de sanción penal que, como su nombre lo indica, son diferentes a la pena privativa de libertad y buscan armonizar los objetivos sancionadores de la pena con los fines resocializadores de la misma que se dirigen al delincuente. 
Si bien no existe un catálogo cerrado que enumere las medidas sustitutivas a la pena de privación de la libertad que se pueden utilizar, las Reglas de Tokio enuncian una serie de medidas sustitutivas, sin perjuicio de que los diferentes Estados diseñen y ejecuten otro tipo de medidas, de acuerdo a sus necesidades sociales y políticas o a la naturaleza y gravedad del delito y personalidad del delincuente. Algunas de las medidas sustitutivas que se proponen en las Reglas de Tokio son: penas privativas de derechos o inhabilitaciones, sanciones económicas y penas en dinero, restitución o indemnización a la víctima, suspensión de la sentencia o condena diferida, imposición de servicios a la comunidad, obligación de acudir regularmente a un centro determinado, arresto domiciliario, entre otras.

En un Estado democrático de derecho, el respeto por los derechos humanos constituye un pilar fundamental, por lo tanto, es obligación del Estado abstenerse de intervenir arbitraria e innecesariamente en los derechos y libertades de los ciudadanos, así como garantizar su plena efectividad. Ahora bien, si tenemos presente la gran importancia que tiene el derecho a la libertad personal dentro de los derechos civiles y políticos y su reconocimiento en los distintos instrumentos internacionales de derechos humanos, es necesario reconocer que cualquier restricción o privación a la libertad deberá fundarse en motivos previamente establecidos en la ley y solo procederá cuando sea absolutamente necesaria.

En el ámbito internacional de los derechos humanos se han adoptado por los organismos de la OEA y de la ONU diversos instrumentos orientados a impulsar la adopción de medidas sustitutivas a la privación de la libertad personal.

Dentro de la OEA tenemos los Principios y Buenas Prácticas sobre la Protección de las Personas Privadas de la Libertad en las Américas, adoptado por la CIDH mediante la Resolución 1 de 2008, que en el principio III, numeral 2, "Excepcionalidad de la privación preventiva de la libertad", y numeral 4, "Medidas alternativas o sustitutivas a la privación de la libertad", se refieren a la materia. Asimismo, en las Recomendaciones de la Segunda Reunión de Autoridades Responsables de las Políticas Penitenciarias y Carcelarias de los Estados Miembros de la OEA se hace una breve alusión al tema concernido.

En el seno de la ONU encontramos (1) las Reglas mínimas de las Naciones Unidas sobre las medidas no privativas de la libertad, conocidas también como Reglas de Tokio; (2) el Informe sobre Medias Sustitutivas de la Prisión y Medidas de Reinserción Social de los Presos, presentada en 1985 en el Séptimo Congreso de la ONU sobre Prevención del Delito y Tratamiento del Delincuente; (3) y los documentos elaborados por la Oficina de las Naciones Unidas contra la Droga y el Delito.

Como vemos, si bien no son abundantes los instrumentos internacionales que se ocupan del tema de medidas sustitutivas a la pena de privación de la libertad, sí existen unas directrices y recomendaciones con carácter vinculante que deben observar los Estados al formular sus políticas criminales en orden a adoptar medidas alternativas para sancionar la 
comisión de delitos, a la par que se promueva la despenalización de ciertas conductas en las que no sea necesaria la intervención del sistema penal. Al respecto, en el numeral 2.6 de los Reglas de Tokio se lee: "La utilización de medidas no privativas de la libertad será parte de un movimiento en pro de la despenalización y destipificación de delitos, y no estarán encaminadas a obstaculizar ni a diferir las iniciativas en ese sentido”.

Teniendo presente los principios de proporcionalidad y necesidad de la pena cuando en el ordenamiento criminal existan otras medidas que no impliquen restricciones a la libertad de las personas y por medio de los cuales se satisfagan los fines de la protección de la sociedad, la resocialización de la persona condenada y la garantía de los derechos de las víctimas, se deberá optar por las medidas sustitutivas que resultan más garantistas de los derechos humanos de las personas condenadas y torna innecesaria la privación de la libertad.

Esta orientación humanista y garantista de los derechos humanos de las personas penadas, configura un importante elemento de distinción entre un Estado autoritario y un Estado democrático, pues mientras el primero usa su poder punitivo como primera medida para reprimir conductas delictuosas, el segundo se asegura de que el ius puniendi y las penas privativas de la libertad se utilicen solo como último recurso, después de que quede plenamente establecido que el uso de otros mecanismos resultan insuficientes para sancionar las conductas delictivas más graves que afecten bienes jurídicos de la más alta importancia.

La jurisprudencia del sistema interamericano se ha referido especialmente al tema de medidas sustitutivas a la pena de privación de la libertad, en relación a los menores de edad, tomando en consideración los diferentes instrumentos internacionales que se refieren a la materia. Por ejemplo, el Convenio sobre los Derechos del Niño en su artículo 40.4 indica: "Se dispondrá de diversas medidas, tales como el cuidado, las órdenes de orientación y supervisión, el asesoramiento, la libertad vigilada, la colocación en hogares de guarda, los programas de enseñanza y formación profesional, así como otras posibilidades alternativas a la internación en instituciones, para asegurar que los nińos sean tratados de manera apropiada para su bienestar y que guarde proporción tanto con sus circunstancias como con la infracción”.

Así entonces, en el Informe No 41/99 sobre menores detenidos en Honduras por situaciones no tipificadas como delitos, tales como vagancia y orfandad, la CIDH precisó que los Estados no pueden utilizar el ius puniendi para abordar los problemas sociales de los niños, pues los métodos sancionatorios deben ser el último recurso estatal para enfrentar los más graves hechos de criminalidad, y señaló: "El derecho internacional de los derechos humanos se dirige a procurar que las penas que imponen graves restricciones de los derechos fundamentales de los menores, sean limitadas únicamente a las infracciones más severas. Por tanto, aun en el caso de infracciones tipificadas, la legislación tutelar del menor debe propender hacia formas de sanción distintas a la reclusión o privación de libertad”. De esta manera la $\mathrm{CIDH}$, por regla general, no considera necesarias las penas privativas de libertad 
sobre menores, dado que existen medidas alternativas como serían por ejemplo las medidas pedagógicas que pueden cumplir efectivamente una función de resocialización y rehabilitación sin impactar negativamente sobre los derechos de los niños.

Ahora bien, aunado a lo anterior, podemos exponer algunas justificaciones políticas o prácticas que nos lleven a replantear el uso de la pena privativa de la libertad como pena principal para sancionar toda clase de delitos comunes.

En efecto, existen varias razones por las cuales se puede afirmar que la pena privativa de la libertad no es la pena más idónea. En primer lugar, se pueden esgrimir razones económicas, ya que los costos de mantener a una persona privada de la libertad son bien altos, pues se necesita construir centros de detención, se debe contar con una gran burocracia estatal que administre y se encargue de estos asuntos, y por supuesto, el Estado debe asumir los costos de alimentación, salud y demás gastos que demanda el cuidado de una persona privada de la libertad.

En segundo lugar, es discutible que las penas privativas de la libertad ayuden a cumplir los fines resocializadores de la pena. En efecto, no existe ningún estudio que muestre que las penas privativas de la libertad son más efectivas que las penas alternativas para resocializar a las personas y evitar que delincan en el futuro, por el contrario, es más difícil para una persona que ha estado privada de la libertad reintegrarse a la sociedad que una que cumple una pena alternativa y se le permite tener contacto con la sociedad y la familia, facilitándole su readaptación.

En tercer lugar, se puede argumentar que las penas de prisión no ayudan ni a prevenir ni a reducir el delito, pues cada día es más elevado el número de personas detenidas, sin embargo, las tasas de delincuencia no parecen disminuir.

Finalmente, el uso generalizado de la pena privativa de la libertad en todo el mundo, el aumento en la duración de las penas y la penalización de conductas que anteriormente no eran delictivas, ha llevado a que la población carcelaria aumente constantemente a nivel mundial. Estas situaciones han llevado a niveles alarmantes de hacinamiento carcelario en muchos países, especialmente en Latinoamérica, lo que genera una violación sistemática de los derechos de las personas privadas de la libertad, puesto que estas situaciones traen como consecuencia problemas de salud, violencia, violaciones a la integridad física y dignidad humana de los reclusos, ya que pueden constituir una forma de trato cruel, inhumano y degradante.

Aunado a lo anterior, es importante destacar que el mayor porcentaje de personas privadas de la libertad se encuentran bajo esta condición por la comisión de delitos menores o en estado de detención preventiva a la espera de una sentencia. Es por esto importante implementar medidas alternativas a la privación de la libertad que reduzcan el hacinamiento 
carcelario, contribuyendo así a garantizar los derechos de las personas privadas de la libertad, pues estas medidas son menos lesivas que la prisión para las personas que buscan resocializarse, al mismo tiempo que implementadas apropiadamente, analizando cada caso en particular, ayudan a cumplir los objetivos sancionadores y de preservación de la seguridad pública.

\section{CONCLUSIÓN}

Como ya hemos mencionado, las modernas corrientes jurídica de derecho penal abogan por el "derecho penal mínimo", es decir, el empleo restrictivo y marginal o ultima ratio del poder punitivo estatal. Sin embargo, contrario a estas corrientes jurídicas, hoy en día es común encontrarnos con reformas en los diferentes sistemas penales nacionales que endurecen las penas, penalizan conductas o restringen los beneficios penales que se les pueden otorgar a las personas condenadas que cumplen con determinados requisitos.

Por lo tanto, de lo expuesto anteriormente, es necesario concluir que siendo la pena privativa de la libertad una medida marginal que solo debe utilizarse cuando sea estrictamente necesaria en vista de la naturaleza y gravedad del delito y la personalidad del delincuente, se deberá preferir entonces la adopción de medidas alternativas o sustitutivas a la pena de privación de la libertad que resultan menos restrictivas de los derechos de las personas condenadas penalmente, y que diseñadas y aplicadas apropiadamente cumplen con los fines del juico penal y las sanciones, a la par que fomenta la readaptación del condenado a la vida en libertad en la sociedad.

Un sistema penal respetuoso de la dignidad humana, enfocado en cumplir no solamente con los fines sancionadores de la pena, sino también con los fines resocializadores de la misma, debería diseñar y adoptar medidas que sustituyan la pena privativa de la libertad en los casos en que resulte manifiestamente innecesaria, profundizando así el carácter protagónico de los derechos humanos en una sociedad democrática, en donde los derechos y libertades de todos los ciudadanos sean considerados sin discriminación alguna y se visibilice y tome conciencia del drama carcelario que viven muchos países del mundo, en donde el hacinamiento, las precarias condiciones de higiene y salubridad en los centros penitenciarios y la violencia al interior de los mismos están a la orden del día.

Ahora bien, no obstante las bondades de las medidas alternativas o sustitutivas a la privación de la libertad y su discusión desde hace varios años en los distintos foros internacionales, aún no ha sido posible implementar una estrategia coherente tendiente a diseñar e implementar dichas medidas, especialmente en nuestros países en donde se acostumbra a manejar problemas de orden público o criminalidad con el endurecimiento de las penas privativas de la libertad y la penalización de conductas. 
Por lo tanto, es necesario que los distintos sectores de la sociedad y el Estado se involucren en el diseńo y puesta en práctica de un sistema de medidas alternativas a la pena privativa de la libertad, ya que para este fin es necesario el apoyo del Poder Legislativo para implementar modificaciones en los ordenamientos penales nacionales, la adopción de tales medidas por parte de los jueces y tribunales, y la concientización de la sociedad en general de que se puede acudir a medidas alternativas para enfrentar conductas criminales sin que se ponga en riesgo la seguridad pública o se trate de una forma encubierta de impunidad. 